\title{
The Development of Biological Molecular Sensing Techniques to detect Micro particles: Focus on Clinical Medicine Benefits
}

\section{Alexander E Berezin*}

Department of Internal Medicine, State Medical University 26, Mayakovsky av, Zaporozhye, Ukraine

\begin{abstract}
Microparticles (MPs) are defined a heterogeneous population of vesicles (diameter 100-1000 nm) that are released by cellular vesiculation and fission of the membrane of cells and play a pivotal role in various diseases including cardiovascular diseases, cancer, sepsis, eclampsia, autoimmune and metabolic states. Currently there is no standardization regarding analytical methods of MP detection. Conventional methods have crucial limitations regarding complicated assay and suffers from relatively low sensitivity and accuracy because of resolution problems occurring for the majority of commercially available flow cytometers. Alternatively, recently recognized as a method for quantification and sizing of biological nanoparticles surface plasmon resonance-based imaging microscopy (SPRi microscopy) might be significantly useful to resolve the majority problems affected MPs recognition. Probably Raman micro-spectroscopy, micro nuclear magnetic resonance technique, small-angle X-ray scattering, and anomalous small-angle X-ray scattering might compete with SPRi microscopy and flow cytometery. Sort comment is discussed contemporary approaches regarding novel techniques of microparticle determination, measurements and assay.
\end{abstract}

Keywords: Microparticles; Analytical assay; Flow cytometry; Western blot analysis; Electron microscopy; Surface plasmon resonance-based imaging microscopy; Nuclear magnetic resonance techniques

Developments of technologies that attenuate recognize, determination, and measurements of microparticles (MPs) obtained from various cells appear to be indispensable tool to clinical medicine [1]. Recent investigations have been shown that MPs as derivate of cellular membrane are discussed powerful paracrine regulators of target cell functions affected growth of tissue, reparation, vasculogenesis, inflammation, apoptosis, infection, and malignancy [2-4]. There is large body of evidences regarding association between immune pattern of MPs originated from different cells (endothelial cells, mononuclear, dendritic cells, platelets) and nature evolution of various diseases including cardiovascular diseases, cancer, sepsis, eclampsia, autoimmune and metabolic states, etc. [5-8].

MPs are defined a heterogeneous population of vesicles (diameter $100-1000 \mathrm{~nm}$ ) that are released by cellular vesiculation and fission of the membrane of cells [9]. This mechanism affects genome and may mediate by some triggers [10]. In is well known that MPs appear to be found into circulation in response to many situational changes (physiological conditions, stress) micro environmental stimulation, coagulation / thrombosis, endotoxinemia, endothelial shear stress, activated cells or those undergoing apoptosis, ischemic injury, hypoxia, and malignancy [11-13].

The current stand of knowledge regarding morphology, transcriptomics, and proteomics of circulating MPs is still not fully $[14,15]$. The difficulty of separating MPs realized from other types of cells limits or efforts to extend actual cognitions in features affected biogenesis, secretion, and subsequent biological role of MPs. Therefore, there are no standardized protocols regarding methods of isolation and analysis of MPs [16].

The conventional approach for measuring the MPs is based on commonly used flow cytometry and nanoparticle tracking analysis (NTA), Western blot analysis and electron microscopy, although the definition of MPs using these techniques is still an area of great debate. Unfortunately, all methods have crucial limitations regarding complicated assay and suffers from relatively low sensitivity and accuracy because of resolution problems occurring for the majority of commercially available flow cytometers $[17,18]$. The next serious barrier created surmountable problems for NTA is sizing of small MPs (50 nm and less). However, the utilization of flow cytometers specifically designed for analysis of small-size MPs is probably to provide considerable methodological advantages and should be the preferable options [17]. In addition, problems with concentration limits of NTA measurements restrict the use of this method for clinical samples [18]. Western blot analysis and electron microscopy allow to optionally recognizing MPs depending on determination of different markers, represents a useful tool for examining particles. However, Western blot analysis and electron microscopy require subsequent technical efforts and are much expensive.

Alternatively, recently recognized as a method for quantification and sizing of biological nanoparticles surface plasmon resonance based imaging microscopy (SPRi microscopy) might be significantly useful to resolve the majority problems affected MPs recognition. SPRi is discussed a highly sensitive label-free biochemical surface sensor measurement technique that has only recently been applied to the field of cell-biology. This method is based on phenomenon known as surface plasmon resonance that associates with a high resolute diffraction generated at a thin metal surface [19]. The high contrast in SPR signal between cell edges and substratum facilitates identification of cell edges and segmentation of cell areas [20]. Importantly that several cells, cellular components (i.e., focal adhesions, nucleus, and cellular secretions), viruses, bacteria, micro- and nanoparticles have not just became visible, but they are able to be calculable $[19,20]$.

*Corresponding author: Alexander E Berezin, Consultant of Cardiology Unit, Internal Medicine Department, State Medical University 26, Mayakovsky av, Zaporozhye, Ukraine, Tel: +380612894585; E-mail: dr_berezin@mail.ru

Received July 05, 2015; Accepted July 22, 2015; Published July 29, 2015

Citation: Berezin AE (2015) The Development of Biological Molecular Sensing Techniques to detect Micro particles: Focus on Clinical Medicine Benefits. J Microb Biochem Technol 7:4 236-237. doi:10.4172/1948-5948.1000214

Copyright: @ 2015 Berezin AE. This is an open-access article distributed under the terms of the Creative Commons Attribution License, which permits unrestricted use, distribution, and reproduction in any medium, provided the original author and source are credited. 
As expected, a quantitative interpretation of SPRi imaging might improve resolution of MP determination and allow investigators unprecedented to overcome flow cytometry limitations regarding low detectable small-size MPs [21]. Moreover, simultaneous application of a high-sensitive fluorescent microscopy and SPRi microscopy should enhance the sensitivity and selectivity of a created biosensor platform $[22,23]$. This might have a high value for identification of small-size MPs originated from different cells that were recently determined as debris [24]. Probably small-size MPs derived from apoptotic cells play a pivotal role in tissue injure, inversely MPs secreted activated cells, i.e., mononuclear, endothelial cells, dendritic cells, may have a positive effect on tissue repair and homeostasis [25].

A highly sensitive fluorescent (HSF) microscopy also permits to detect individual sub-micro and nano-MPs. As compared with SPRi microscopy, this technique could provide higher detection sensitivity due to a large fluorescence excitation and a high effective quantum yield of fluorescence. Therefore, there are at least four methods that are not commercially available: Raman micro-spectroscopy, micro nuclear magnetic resonance technique, small-angle X-ray scattering, and anomalous small-angle X-ray scattering [26]. All these methods are currently being explored to assay MPs, while an incorporation of these techniques into routine analytical practice is probably addressed in the future.

In conclusion, a standardization of the methods of nano- and micro- particles determination is extremely required. Commonly used procedures, such as flow cytometry with NTA, Western blot analysis and electron microscopy, might not have universal utility for MP determination; especially for small-size MPs. Novel techniques regarding identification of MPs based on real-time and label-free optical biosensors and principles of SPR phenomena appear to be much attractive and could sufficiently overcome limitation of option methods of MP determination.

\section{References}

1. Gu Z, Jing C, Ying YL, He P3, Long YT2 (2015) In situ high throughput scattering light analysis of single plasmonic nanoparticles in living cells. Theranostics 5 : 188-195

2. Gong J, Jaiswal R, Dalla P, Luk F, Bebawy M (2015) Microparticles in cancer: A review of recent developments and the potential for clinical application. Semin Cell Dev Biol 40: 35-40

3. Das S, Halushka MK (2015) Extracellular vesicle microRNA transfer in cardiovascular disease. Cardiovasc Pathol 24: 199-206.

4. Berezin A, Zulli A, Kerrigan S, Petrovic D, Kruzliak P (2015) Predictive role of circulating endothelial-derived microparticles in cardiovascular diseases. Clin Biochem 48: 562-568.

5. Jadli A, Sharma N, Damania K, Satoskar P, Bansal V, et al. (2015) Promising prognostic markers of Preeclampsia: New avenues in waiting. Thromb Res.

6. Greening DW, Gopal SK, Mathias RA, Liu L, Sheng J, et al. (2015) Emerging roles of exosomes during epithelial-mesenchymal transition and cancer progression. Semin Cell Dev Biol 40: 60-71.

7. Martinez MC, Andriantsitohaina R (2011) Microparticles in angiogenesis: Therapeutic potential. Circ Res 109: 110-119.

8. Souza AC, Yuen PS, Star RA (2015) Microparticles: Markers and mediators of sepsis-induced microvascular dysfunction, immunosuppression, and AKI. Kidney Int 87: 1100-1108.

9. Colombo M, Raposo G, Théry C (2014) Biogenesis, secretion, and intercellular interactions of exosomes and other extracellular vesicles. Annu Rev Cell Dev Biol 30: 255-289.

10. Piccin A, Murphy WG, Smith OP (2007) Circulating microparticles: Pathophysiology and clinical implications. Blood Rev 21: 157-171.

11. Lynch SF, Ludlam CA (2007) Plasma microparticles and vascular disorders. Br J Haematol 137: 36-48.

12. Sabatier F, Camoin-Jau L, Anfosso F, Sampol J, Dignat-George F (2009) Circulating endothelial cells, microparticles and progenitors: Key players towards the definition of vascular competence. J Cell Mol Med 13: 454-471.

13. Burnier L, Fontana P, Kwak BR, Angelillo-Scherrer A (2009) Cell-derived microparticles in haemostasis and vascular medicine. Thromb Haemost 101: 439-451.

14. Inal JM, Kosgodage U, Azam S, Stratton D, Antwi-Baffour S, et al. (2013) Blood/ plasma secretome and microvesicles. Biochim Biophys Acta 1834: 2317-2325.

15. Choi DS, Kim DK, Kim YK, Gho YS (2013) Proteomics, transcriptomics and lipidomics of exosomes and ectosomes. Proteomics 13: 1554-1571.

16. Dinkla S, Brock R, Joosten I, Bosman GJ (2013) Gateway to understanding microparticles: Standardized isolation and identification of plasma membranederived vesicles. Nanomedicine (Lond) 8: 1657-1668.

17. Shantsila E, Montoro-García S, Gallego P, Lip GY (2014) Circulating microparticles: Challenges and perspectives of flow cytometric assessment. Thromb Haemost 111: 1009-1014.

18. Orozco AF, Lewis DE (2010) Flow cytometric analysis of circulating microparticles in plasma. Cytometry A 77: 502-514.

19. Peterson AW, Halter M, Tona A, Plant AL (2014) High resolution surface plasmon resonance imaging for single cells. BMC Cell Biol 15: 35.

20. Peterson AW, Halter M, Tona A, Bhadriraju K, Plant AL (2010) Using surface plasmon resonance imaging to probe dynamic interactions between cells and extracellular matrix. Cytometry A. 77: 895-903.

21. Robelek R, Wegener J (2010) Label-free and time-resolved measurements of cell volume changes by surface plasmon resonance (SPR) spectroscopy. Biosens Bioelectron 25: 1221-1224.

22. Shpacovitch V (2012) Application of Surface Plasmon Resonance (SPR) for the Detection of Single Viruses and Single Biological Nano-objects. J Bacteriology and Parasitology 3: e110.

23. Zybin A, Kuritsyn YA, Gurevich EL, Temchura VV, Überla K, et al. (2010) Real-time detection of single immobilized nanoparticles by surface plasmon resonance imaging. Plasmonics 5: 31-35.

24. Berezin AE, Kremzer AA, Samura TA, Berezina TA, Kruzliak P (2015) Impaired immune phenotype of circulating endothelial-derived microparticles in patients with metabolic syndrome and diabetes mellitus. J Endocrinol Invest 38: 865874

25. Berezin AE (2015) Impaired Phenotype of Circulating Endothelial-Derived Microparticles: Novel Marker of Cardiovascular Risk. J Cardiology and Therapy 2: 273-278.

26. van der Pol E, Coumans F, Varga Z, Krumrey M, Nieuwland R (2013) Innovation in detection of microparticles and exosomes. J Thromb Haemost 11 Suppl 1 : $36-45$ 